\title{
A Combined Weighting Method Based on Hybrid of Interval Evidence Fusion and Random Sampling
}

\author{
Ying Yan ${ }^{1}$ and Bin Suo ${ }^{2}$ \\ ${ }^{1}$ School of Economics and Management, Southwest University of Science and Technology, Mianyang 621010, China \\ ${ }^{2}$ Institute of Electronic Engineering, China Academy of Engineering Physics, Mianyang 621900, China \\ Correspondence should be addressed to Ying Yan; yanying_527@163.com
}

Received 2 November 2016; Revised 25 January 2017; Accepted 15 February 2017; Published 20 March 2017

Academic Editor: Ricardo López-Ruiz

Copyright (c) 2017 Ying Yan and Bin Suo. This is an open access article distributed under the Creative Commons Attribution License, which permits unrestricted use, distribution, and reproduction in any medium, provided the original work is properly cited.

\begin{abstract}
Due to the complexity of system and lack of expertise, epistemic uncertainties may present in the experts' judgment on the importance of certain indices during group decision-making. A novel combination weighting method is proposed to solve the index weighting problem when various uncertainties are present in expert comments. Based on the idea of evidence theory, various types of uncertain evaluation information are uniformly expressed through interval evidence structures. Similarity matrix between interval evidences is constructed, and expert's information is fused. Comment grades are quantified using the interval number, and cumulative probability function for evaluating the importance of indices is constructed based on the fused information. Finally, index weights are obtained by Monte Carlo random sampling. The method can process expert's information with varying degrees of uncertainties, which possesses good compatibility. Difficulty in effectively fusing high-conflict group decision-making information and large information loss after fusion is avertible. Original expert judgments are retained rather objectively throughout the processing procedure. Cumulative probability function constructing and random sampling processes do not require any human intervention or judgment. It can be implemented by computer programs easily, thus having an apparent advantage in evaluation practices of fairly huge index systems.
\end{abstract}

\section{Introduction}

The establishment of index system and the determination of corresponding index weights are very significant during the appraisal processes of risk assessment, system evaluation, and economic efficiency evaluation. Considering the important role of weight during the appraisal process that reflects the effect of the index on the previous level decision-making elements, the accuracy of evaluation and the correctness of decision-making are all based on it. The index weighting method can be classified into subjective weighting method, objective weighting method, and combination weighting method due to the diversity of data sources.

Subjective weighting refers to the method in which the importance of indices was assessed according to the qualitative judgment of the decision-makers based on their professional knowledge and experience. And the index weights of these judgments were quantified through some specific standard. Common subjective weighting methods include cyclic scoring, AHP, FAHP, and binomial coefficient method. AHP is the most widely used one among them. However, it is difficult to achieve construction or examination of consistent judgment matrices for AHP. The consistency check of multiexpert group weighting problems remains difficult despite the fact that the difficulty in constructing consistent judgment matrices can be solved by a group order relation analysis for AHP. In general, subjective weighting methods have not advanced sufficiently.

The weights were determined according to the relationship between the original data in objective weighting method. Typical objective weighting methods include entropy method, multiobjective programming, and principal component analysis. Compared with subjective weighting methods, objective weighting methods own better 
TABLE 1: Sample table of questionnaire.

\begin{tabular}{|c|c|c|c|c|c|}
\hline & Not at all important & Unimportant & Moderately important & Important & Very important \\
\hline Expert 1 & & $\begin{array}{c}\sqrt{ } \\
0.6\end{array}$ & $\begin{array}{c}\sqrt{ } \\
0.3\end{array}$ & $\begin{array}{c}\sqrt{ } \\
0.1\end{array}$ & \\
\hline Expert 2 & $\begin{array}{c}\sqrt{ } \\
{[0.1,0.3]}\end{array}$ & $\begin{array}{c}\sqrt{ } \\
{[0.6,0.9]}\end{array}$ & & & \\
\hline Expert 3 & & $\begin{array}{c}\sqrt{ } \\
1.0\end{array}$ & & & \\
\hline
\end{tabular}

Weights of different indices can be obtained by synthesizing evaluation opinions of $L$ number of experts.

mathematical bases but worse interpretability. Thus, their conclusions are inconsistent with the actual importance of indices at times [1].

To overcome the shortcomings of the subjective and objective weighting methods, in recent years, methods which combine the subjective and objective weighting have been developing rapidly. These methods include modified AHP and the entropy method [2], maximum entropy weighting [3], geometric operator weighting [4], and weighting method based on maximizing the difference in evaluation results [5]. But in general, most of the computing methods are complicated, and some of them also can hardly reflect different emphasis of experts placed on different indices. Thus, they are still imperfect. A variance maximization-based combination weighting method was proposed by Sun and Bao [6]. It determined the weights of group decision-making information by using AHP and rough set theory respectively. Then the comprehensive weights were obtained by variance maximization of the two previous weights. The method is rational to some extent. However, construction of judgment matrix and consistency check for group decision-making information remains difficult in case of highly conflicting opinions. A structure entropy method was proposed by Cheng [7]. In this manner, the qualitative "typical ranking" was replaced by a quantitative membership function through entropy function before postprocessing. Although the method can achieve good results, it is difficult for experts to reasonably rank the importance of numerous indices for the relatively large index system. Three different secondary sorting methods were used to rank evaluation indices; rational ranking would be obtained by Kendall test when the previous ranking is contradictory [8]. Combined weights of indices were obtained ultimately by calculating the standard deviations of evaluation indices. The method can effectively reduce the inconsistencies in importance ranking. Nevertheless, the subjective determination of reasonable ranking and the importance ratios of indices can still exist. In addition, due to the complexity of system and lack of expertise, epistemic uncertainties may occur in judgment of group decision-making for the importance of certain indices [9]. For example, they may be unable to fully determine whether a certain index is "important" or "very important". Currently, researches on index weighting determination during expert evaluation under epistemic uncertainties are still insufficient.

Accordingly, in order to solve the above problems, various types of uncertain information of expert comments during group decision-making were fully considered in the paper. A unified expression is proposed for this uncertain information based on the reconstruction of interval evidence structure. And multisource information is fused according to the similarity of interval evidences. Then, cumulative probability function is constructed based on this to evaluate the importance of indices. Finally, index weights are determined by Monte Carlo random sampling.

\section{Problem Description}

Assume that the number of experts involved in evaluating a certain project is $L$. Experts set was denoted as $\mathbf{E}=$ $\left\{e_{1}, e_{2}, \ldots, e_{L}\right\}$; the number of indices involved in the project is $M$. The index set was denoted as $\mathbf{A}=\left\{a_{1}, a_{2}, \ldots, a_{M}\right\}$. In general, it was difficult for experts to directly determine the weight of each index. And evaluating the importance degree of indices by comment grades as "not at all important," "unimportant," "moderately important," "important," and "very important" was comparatively easy. Comment grade set was denoted as $\mathbf{H}=\left\{H_{1}, H_{2}, H_{3}, H_{4}, H_{5}\right\}$. Evaluation opinions of expert $e_{i}(i=1,2, \ldots, L)$ 's on index $a_{k}(k=$ $1,2, \ldots, M)$ were

$$
C_{i}\left(a_{k}\right)=\left\{H_{j}, p_{i, j}\left(a_{k}\right) \mid j=1,2, \ldots, 5\right\},
$$

where $p_{i, j}\left(a_{k}\right)$ was the credibility of $e_{i}$ 's evaluation for index $a_{k}$ by comment grade $H_{j}$. Considering the epistemic uncertainties, experts were allowed to select 1 to 3 comment grades for the same index in the questionnaire. $p_{i, j}\left(a_{k}\right)$ could be either point value or interval number. Table 1 is an example of questionnaires. Obviously, $p_{i, j}\left(a_{k}\right)$ satisfied the following equation:

$$
\begin{aligned}
0 & \leq p_{i, j}\left(a_{k}\right) \leq 1, \\
\min \sum_{j=1}^{N} p_{i, j}\left(a_{k}\right) & \leq 1, \\
\max \sum_{j=1}^{N} p_{i, j}\left(a_{k}\right) & \geq 1 .
\end{aligned}
$$

\section{Novel Weighting Method}

Due to the epistemic uncertainties of experts and inevitable inconsistencies in different experts' opinions on the same 
index, how to rationally fuse expert information was a very critical issue. Evidence theory is a potent method to fuse multisource uncertain information, which has been widely used in the fields of data fusion, artificial intelligence, expert system, and decision analysis [10-12]. To get more reasonable and reliable fusion results, the expression form and idea of interval evidence theory were utilized to fuse expert's comments in this paper. Finally, the index weights were determined by random sampling of fused information.

3.1. Interval Evidence Theory. Interval evidence theory is established based on the frame of discernment. The frame of discernment is defined as the set containing all possible results for a specific problem. It is generally a nonempty set and represented by $\Theta$.

Definition 1. Interval basic probability assignment (IBPA) [12-15]: let $m$ be a mapping from the power set to the interval number $[0,1]$; that is, $m: 2^{\Theta} \rightarrow[0,1]$, for $\Theta$ 's $n$ number of subsets $A_{i}(i=1,2, \ldots, n)$; IBPA was

$$
m\left(A_{i}\right)=\left[a_{i}, b_{i}\right]
$$

where $0 \leq a_{i} \leq b_{i} \leq 1$. If IBPA satisfied simultaneously the following conditions

$$
\begin{aligned}
a_{i} & \leq m\left(A_{i}\right) \leq b_{i}, \\
\sum_{i=1}^{n} a_{i} & \leq 1, \\
\sum_{i=1}^{n} b_{i} & \geq 1, \\
m(H) & =0, \quad \forall H \notin \Theta
\end{aligned}
$$

then $m$ was called a valid IBPA.

Definition 2. Normalization principle $[15,16]$ : if $m$ satisfied (4), which was a valid IBPA, and $m\left(A_{i}\right)=\left[a_{i}, b_{i}\right]$ (where $\left.0 \leq a_{i} \leq b_{i} \leq 1\right)$ and if $a_{i}$ and $b_{i}$ satisfied simultaneously the following conditions

$$
\begin{aligned}
& \sum_{j=1}^{n} b_{j}-\left(b_{i}-a_{i}\right) \geq 1, \\
& \sum_{j=1}^{n} a_{j}+\left(b_{i}-a_{i}\right) \geq 1,
\end{aligned}
$$

$$
(i, j=1,2, \ldots, n)
$$

then $m$ was called normalized IBPA.

If $m$ was a valid IBPA but not normalized, normalization would be needed according to the formula below in order to narrow the interval width and reduce redundancy:

$$
\begin{gathered}
\max \left[a_{i}, 1-\sum_{j=1}^{n} b_{j \neq 1}\right] \leq m\left(A_{i}\right) \\
\leq \min \left[b_{i}, 1-\sum_{j=1}^{n} a_{j}\right] .
\end{gathered}
$$

Definition 3. Dempster's rule of interval evidence combination $[15,16]$ : wvidences from different information sources could be fused into more reliable, accurate information using the rule of evidence combination. As the most classic evidence combination rule, Dempster's rule could be extended to the field of interval evidence. Let $m_{1}$ and $m_{2}$ be the valid, normalized IBPA under the same frame of discernment $\Theta$, where

$$
\begin{array}{ll}
m_{1}\left(A_{i}\right)=\left[a_{i}, b_{i}\right] \quad\left(0 \leq a_{i} \leq b_{i} \geq 1, i=1,2, \ldots, n\right), \\
m_{2}\left(A_{j}\right)=\left[a_{j}, b_{j}\right] & \\
& \left(0 \leq a_{j} \leq b_{j} \geq 1, j=1,2, \ldots, n\right) .
\end{array}
$$

Then Dempster's rule of interval evidence combination was defined as

$$
\begin{aligned}
& {\left[m_{1} \oplus m_{2}\right](C)} \\
& \quad= \begin{cases}\left(\left(m_{1} \oplus m_{2}\right)^{+}(C),\left(m_{1} \oplus m_{2}\right)^{-}(C)\right) & C \neq \varnothing \\
0 & C=\varnothing,\end{cases}
\end{aligned}
$$

where $\left(m_{1} \oplus m_{2}\right)^{+}(C)$ and $\left(m_{1} \oplus m_{2}\right)^{-}(C)$ were the maximum and minimum values, respectively, in the formula below:

$$
\begin{array}{ll}
\max / \min \quad & {\left[m_{1} \oplus m_{2}\right](C)} \\
& =\frac{\sum_{A_{i} \cap A_{j}=C} m_{1}\left(A_{i}\right) m_{2}\left(A_{j}\right)}{1-\sum_{A_{i} \cap A_{j}=\varnothing} m_{1}\left(A_{i}\right) m_{2}\left(A_{j}\right)} \\
\text { s.t. } \quad & \sum_{i=1}^{n} m_{1}\left(A_{i}\right)=1, \\
& a_{i} \leq m_{1}\left(A_{i}\right) \leq b_{i}, i=1,2, \ldots, n \\
& \sum_{j=1}^{n} m_{2}\left(A_{j}\right)=1, \\
a_{j} \leq m_{2}\left(A_{j}\right) \leq b_{j}, j=1,2, \ldots, n .
\end{array}
$$

As indicted in (9), traversal of all point values BPA (basic probability assignment) satisfying constraints in the IBPA interval was needed during the combination of interval evidences to form fused point value sets. Final combination results could be obtained by taking the maximum and minimum values in these point value sets. 
3.2. Fusion of Group Decision-Making Information. L number of experts' comments on indices $a_{k}(k=1,2, \ldots, M)$ in a certain project could be written as

$$
\mathbf{C}^{\mathbf{k}}=\left[\begin{array}{c}
C_{1}\left(a_{k}\right) \\
C_{2}\left(a_{k}\right) \\
\vdots \\
C_{L}\left(a_{k}\right)
\end{array}\right]
$$

Its IBPA matrix was

$$
\mathbf{m}\left(\mathbf{C}^{\mathbf{k}}\right)=\left[\begin{array}{cccc}
p_{1,1}\left(a_{k}\right) & p_{1,2}\left(a_{k}\right) & \cdots & p_{1,5}\left(a_{k}\right) \\
p_{2,1}\left(a_{k}\right) & p_{2,2}\left(a_{k}\right) & \cdots & p_{2,5}\left(a_{k}\right) \\
\vdots & \vdots & \vdots & \vdots \\
p_{L, 1}\left(a_{k}\right) & p_{L, 2}\left(a_{k}\right) & \cdots & p_{L, 5}\left(a_{k}\right)
\end{array}\right] .
$$

Inconsistencies in different experts' opinions on index $a_{k}$ were inevitable. Thus, conflict handling during the opinions fusion process was very critical. Based on the principle that the greater the conflict with group opinions, the lower the credibility, expert opinions should be fused after assignment of certain weights to obtain reliable results. Similarity between evidences reflects conflicting opinions from another aspect. Thus, similarity between expert opinions was calculated first. Weights of expert comments were then calculated based on the calculated similarity.

Let the frame of discernment for expert evaluation opinions be $\Theta$ and IBPA of experts $e_{n}(1 \leq n \leq L)$ and $e_{r}(1 \leq r \leq L)$ 's comments on index $a_{k}$ be $m_{n}^{k}$ and $m_{r}^{k}$, respectively. Then the Euclidean distance between experts $e_{n}$ and $e_{r}$ 's comments on index $a_{k}$ was defined as

$$
d_{n, r}^{k}=\sqrt{\frac{1}{2 N} \sum_{i=1 n}^{N}\left(\left(\inf \left(m_{n}^{k}\left(H_{i}\right)\right)-\inf \left(m_{r}^{k}\left(H_{i}\right)\right)\right)^{2}+\left(\sup \left(m_{n}^{k}\left(H_{i}\right)\right)-\sup \left(m_{r}^{k}\left(H_{i}\right)\right)\right)^{2}\right)},
$$

where $m_{n}^{k}\left(H_{i}\right)$ and $m_{r}^{k}\left(H_{i}\right)$ were the interval numbers, $\inf (\cdot)$ was their lower bound, and sup(·) was their upper bound.

Distance matrix for pairwise evidences could be obtained by (12) as

$$
D^{k}=\left[\begin{array}{cccc}
0 & d_{12}^{k} & \cdots & d_{1 L}^{k} \\
d_{21}^{k} & 0 & \cdots & d_{2 L}^{k} \\
\vdots & \vdots & & \vdots \\
d_{L 1}^{k} & d_{L 2}^{k} & \cdots & 0
\end{array}\right]
$$

Similarity between experts $e_{n}$ and $e_{r}$ 's comments on index $a_{k}$ could then be obtained as

$$
s_{n r}^{k}=1-d_{n r}^{k} .
$$

Accordingly, similarity matrix for $L$ number of experts' comments on index $a_{k}$ could be obtained as

$$
\mathbf{S}^{\mathbf{k}}=\left[\begin{array}{cccc}
1 & S_{1,2}^{k} & \cdots & S_{1, L}^{k} \\
S_{2,1}^{k} & 1 & \cdots & S_{2, L}^{k} \\
\vdots & \vdots & \vdots & \vdots \\
S_{L, 1}^{k} & S_{L, 2}^{k} & \cdots & 1
\end{array}\right]
$$

Various rows of $\mathbf{S}^{\mathbf{k}}$ were added together to obtain various experts' degrees of support to comment $C_{i}\left(a_{k}\right)$ as

$$
z_{i}^{k}=\sum_{n=1}^{L} S_{n, r}^{k} \quad(i, n=1,2, \ldots, L) .
$$

Weight of comment $C_{i}\left(a_{k}\right)$ could be obtained by normalization as

$$
w_{i}^{k}=\frac{z_{i}^{k}}{\sum_{i=1}^{L} z_{i}^{k}} \quad(i=1,2, \ldots, L) .
$$

BPA matrix in (11) was processed by weighted mean method with $w_{i}^{k}$ as the weight

$$
\begin{aligned}
\widetilde{\mathbf{m}}\left(\mathbf{C}^{\mathbf{k}}\right) & =\left[\begin{array}{llll}
w_{1}^{k} & w_{2}^{k} & \cdots & w_{L}^{k}
\end{array}\right] \times \mathbf{m}\left(\mathbf{C}^{\mathbf{k}}\right) \\
& =\left[\widetilde{m}_{k}\left(H_{1}\right), \ldots, \widetilde{m}_{k}\left(H_{j}\right), \ldots, \widetilde{m}_{k}\left(H_{5}\right)\right] .
\end{aligned}
$$

Equation (18) was precisely the final fusion results of expert evaluation information.

It was noteworthy that, during the information fusion process, we did not use the approach mentioned in literatures [14-16] that the weighted average evidences were recombined $L-1$ times applying Dempster's rule. This was because that the self-combination $L-1$ times of weighted average evidences would induce apparent "focusing" effect which means the fusion results would converge fast towards to focal elements of larger BPA. The fast convergence was helpful to target identification and fusion decision. However, it was unsuitable for objective description of multiple experts' evaluation opinions and results in the high probability of biased results due to the strong subjective tendency and unreasonable explanation of the operation. For example, suppose that an index was commented by 10 experts and weighted average of expert group's opinions was

$$
\widetilde{\mathbf{m}}=\left[\begin{array}{lllll}
0.42 & 0.52 & 0.06 & 0 & 0
\end{array}\right] .
$$

After recombining 9 times of evidence by Dempster's rule, the combined expert opinions could be obtained as

$$
\widehat{\mathbf{m}}=\left[\begin{array}{lllll}
0.18 & 0.82 & 0 & 0 & 0
\end{array}\right] \text {. }
$$


It can be seen that focusing effect was very apparent after combination. After weighted averaging operation without the recombination, the confidence probability of the index which was displayed as "very important" or "unimportant" was high. However, after $L-1$ times of combination, the confidence probability of "very important" was almost completely abandoned, while the confidence probability of "unimportant" was greatly enhanced. Obviously, the results after 9 times of self-combination were far deviated from the original determinations of experts, which would affect the subsequent judgments greatly. Therefore, Dempster's rule was not used in this paper for self-combination of weighted average evidences during the information fusion. We did this way aiming to reflect the experts' original judgments objectively and to utilize known information to the utmost. This could lay a solid foundation for the subsequent objective weighting of indices.

3.3. Random Weight Calculation Method Based on Monte Carlo. Interval numbers $[0,5]$ were divided into five continuous intervals

$$
\begin{aligned}
\mathbf{V} & =\left(V_{1}, V_{2}, \ldots, V_{5}\right) \\
& =([0,1],(1,2],(2,3],(3,4],(4,5])
\end{aligned}
$$

which correspond to the 5 comment grades of the comment set $\mathbf{H}$ and can transform qualitative comments into quantitative values. Clearly

$$
m_{k}\left(V_{j}\right)=\widetilde{m}_{k}\left(H_{j}\right) \quad(j=1,2, \ldots, 5) .
$$

Based on the fusion results of the expert group's opinions, the probability density function (PDF) for importance of index $a_{k}$ was constructed as

$$
f_{k}(x)=\sum_{j=1}^{N} \delta\left(x \mid V_{j}\right) m_{k}\left(V_{j}\right),
$$

where

$$
\delta\left(x \mid V_{j}\right)=\left\{\begin{array}{ll}
1 & \text { if } x \in V_{j} \\
0 & \text { if } x \notin V_{j},
\end{array} \quad 1 \leq j \leq N .\right.
$$

Notably, $m_{k}\left(V_{j}\right)$ in (23) was the interval number, so the cumulative probability function for importance of index $a_{k}$ was in the form of probability envelope. Its lower bound was

$$
\underline{\mathrm{CDF}_{k}}(x)=\int_{0}^{x} \inf \left(f_{k}(t)\right) d t .
$$

And the upper bound was

$$
\overline{\mathrm{CDF}_{k}}(x)=\int_{0}^{x} \sup \left(f_{k}(t)\right) d t .
$$

Mean of the upper and lower bounds was taken as the estimated value of CDF standing for the importance of index $a_{k}$ :

$$
\widehat{F}(x)=\frac{\mathrm{CDF}_{k}(x)+\overline{\mathrm{CDF}_{k}}(x)}{2} .
$$

Meanwhile, it was noted that $m_{k}\left(V_{j}\right)$ was IBPA. Equations (25) and (26) must satisfy $\max \left(\mathrm{CDF}_{k}(x)\right) \leq 1$ and $\max \left(\overline{\mathrm{CDF}_{k}}(x)\right) \leq 1$, so there might be the situation in which $\max (\widehat{F}(x)) \neq 1$. Thus, normalization of (27) was needed:

$$
\widehat{F}(x)=\frac{\widehat{F}(x)}{\max (\widehat{F}(x))} .
$$

Inverse function of (23) was sought

$$
x_{l}^{k}=F_{k}^{-1}(P) .
$$

By Monte Carlo simulation, $N$ number of probability values between $[0,1]$ was randomly generated, and $N$ number of samples $x_{l}^{k}$ was obtained by sampling. Average importance $u_{k}$ of index $a_{k}$ could be obtained by

$$
u_{k}=\frac{\sum_{l=1}^{N} x_{l}^{k}}{N} .
$$

After the normalization of $u_{k}$, the weight of index $a_{k}$ could be obtained as

$$
\omega_{k}=\frac{u_{k}}{\sum_{k=1}^{M} u_{k}} .
$$

\section{Numerical Examples}

A home appliance manufacturer planned to choose a logistics company from 10 candidates as its third-party logistics service provider. The evaluation and choice were carried out from four respective aspects: assets and solvency, profitability, service capability, and innovative development ability. During the evaluation, importance of each index, i.e., index weight, should be determined first. Thus, five peer experts were invited by company A to score the importance of four indices based on the template in Table 1. The results are shown in Table 2.

As can be seen from Table 2, experts' evaluation opinions were largely conflicting. The evaluation information contained multiple types of information such as precise information, epistemic uncertain information, and multi-interval probability information. During the processing, it is needed to express different types of information communications as interval evidence structure. For example, $H_{3}(1)$ could be expressed as $H_{3}([1,1]) ; H_{2}(0.2)$ and $H_{3}(0.8)$ could be expressed as $H_{2}([0.2,0.2]), H_{3}([0.8,0.8])$.

Firstly, each evaluation opinion in Table 2 was checked for whether it was normalized IBPA. For example, for "assets and solvency" index, expert $e_{2}$ 's opinion did not satisfy (3); thus normalization was needed following (4). Normalization results of Table 2 are shown in Table 3.

Afterwards, the evaluation opinions of five experts were fused. For the evaluation index "assets and solvency," 
TABLE 2: Evaluation opinions of experts on the importance of various indices.

\begin{tabular}{ccccc}
\hline & $\begin{array}{c}\text { Assets and } \\
\text { solvency }\end{array}$ & Profitability & Service capability & Innovative development ability \\
\hline$e_{1}$ & $H_{2}(0.2), H_{3}(0.8)$ & $H_{4}(0.7), H_{5}(0.3)$ & $H_{4}(0.1), H_{5}(0.9)$ & $H_{1}(0.8), H_{2}(0.2)$ \\
\hline$e_{2}$ & $\begin{array}{c}H_{1}([0,0.2]), \\
H_{2}([0.2,0.3]), \\
H_{3}([0.7,0.8])\end{array}$ & $H_{2}([0.1,0.3]), H_{3}([0.4,0.5]), H_{4}([0.3,0.6])$ & $H_{4}(0.5), H_{5}(0.5)$ & $H_{1}(1)$ \\
\hline$e_{3}$ & $H_{2}(0.6), H_{3}(0.4)$ & $H_{3}([0.3,0.5]), H_{4}([0.4,0.7])$ & $H_{5}(1)$ & $H_{2}(0.5), H_{3}(0.5)$ \\
\hline$e_{4}$ & $H_{2}(1)$ & $H_{3}(1)$ & $H_{3}(0.2), H_{4}(0.4), H_{5}(0.4)$ & $H_{2}(0.3), H_{3}(0.7)$ \\
\hline$e_{5}$ & $H_{2}([0.2,0.4])$, & $H_{4}([0.2,0.4]), H_{5}([0.5,0.8))$ & $H_{1}(0.2), H_{2}(0.8)$ \\
\hline
\end{tabular}

TABLE 3: Normalized expert's evaluation opinions.

\begin{tabular}{ccccc}
\hline & $\begin{array}{c}\text { Assets and } \\
\text { solvency }\end{array}$ & Profitability & Service capability & $\begin{array}{c}\text { Innovative } \\
\text { development ability }\end{array}$ \\
\hline$e_{1}$ & $H_{2}(0.2), H_{3}(0.8)$ & $H_{4}(0.7), H_{5}(0.3)$ & $H_{4}(0.1), H_{5}(0.9)$ & $H_{1}(0.8), H_{2}(0.2)$ \\
\hline & $H_{1}([0,0.1])$, & $H_{2}([0.1,0.3]), H_{3}([0.4,0.5]), H_{4}([0.3,0.5])$ & $H_{4}(0.5), H_{5}(0.5)$ & \\
$e_{2}$ & $\begin{array}{c}H_{2}([0.2,0.3]), \\
H_{3}([0.7,0.8])\end{array}$ & $H_{3}([0.3,0.5]), H_{4}([0.5,0.7])$ & $H_{1}(1)$ \\
\hline$e_{3}$ & $H_{2}(0.2), H_{3}(0.8)$ & $H_{3}(1)$ & $H_{5}(1)$ & $H_{2}(0.5), H_{3}(0.5)$ \\
\hline$e_{4}$ & $H_{2}(1)$ & $H_{5}(0.2), H_{4}(0.4)$, & $H_{2}(0.3), H_{3}(0.7)$ \\
\hline$e_{5}$ & $H_{2}([0.2,0.4])$, & $H_{4}([0.2,0.4]), H_{5}(0.2)$ & $H_{5}([0.6,0.8))$ & $H_{1}(0.2), H_{2}(0.8)$ \\
\hline
\end{tabular}

Euclidean distance between expert comments was calculated according to (12)-(14), and the result was

$$
D^{k}=\left[\begin{array}{ccccc}
0 & 0.0548 & 0.2530 & 0.5060 & 0.0894 \\
0.0548 & 0 & 0.2258 & 0.4764 & 0.0548 \\
0.2530 & 0.2258 & 0 & 0.2530 & 0.2000 \\
0.5060 & 04764 & 02530 & 0 & 0.4472 \\
0.0894 & 0.0548 & 0.2000 & 0.4472 & 0
\end{array}\right]
$$

Similarity coefficient matrix could thereby be obtained:

$$
S^{k}=\left[\begin{array}{lllll}
1.0000 & 0.9452 & 0.7470 & 0.4940 & 0.9106 \\
0.9452 & 1.0000 & 0.7742 & 0.5236 & 0.9452 \\
0.7470 & 0.7742 & 1.0000 & 0.7470 & 0.8000 \\
0.4940 & 0.5236 & 0.7470 & 1.0000 & 0.5528 \\
0.9106 & 0.9452 & 0.8000 & 0.5528 & 1.0000
\end{array}\right] .
$$

Support degree of each expert's evaluation opinions could be obtained based on (16):

$$
z^{k}=\left[\begin{array}{lllll}
4.0968 & 4.1882 & 4.0682 & 3.3174 & 4.2086
\end{array}\right] .
$$

After normalization, weight coefficient of expert opinions could be obtained:

$$
w^{k}=\left[\begin{array}{lllll}
0.206 & 0.2107 & 0.2046 & 0.1669 & 0.2117
\end{array}\right] .
$$

Expert opinions on the index "assets and solvency" were averaged by weighted means to obtain fused expert opinions:

$$
\widetilde{m}\left(C^{1}\right)=[[0,0.02][0.42,0.49][0.52,0.58][0,0][0,0]]
$$

Based on (23)-(27), CDF for importance of index "assets and solvency" was constructed, and the results are shown in Figure 1.

Similarly, fused expert opinions on the index "profitability" could be obtained as follows:

$$
\widetilde{m}\left(C^{2}\right)=[[0,0][0.02,0.06][0.32,0.38][0.48,0.56][0.10,0.10]]
$$

CDF for its importance is shown in Figure 2.

Fused expert's opinions on the index "service capability" could be obtained as follows:

$$
\widetilde{m}\left(C^{3}\right)=[[0.39,0.39][0.36,0.36][0.24,0.24][0,0][0,0]]
$$

CDF for its importance is shown in Figure 3. 


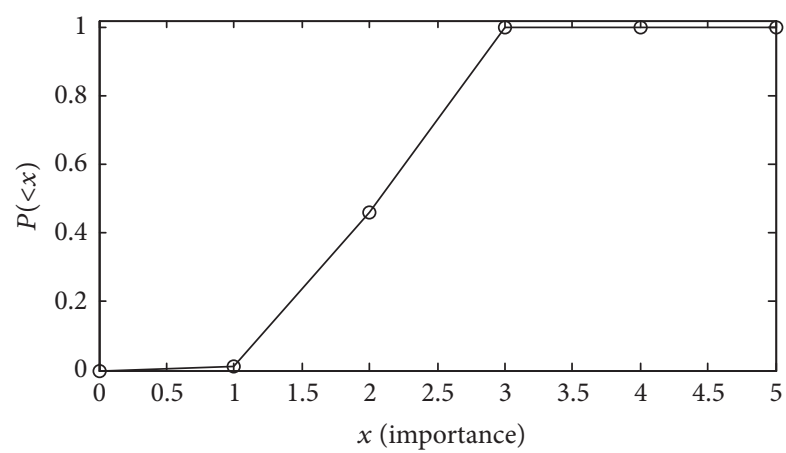

Figure 1: CDF for importance of "assets and solvency" index.

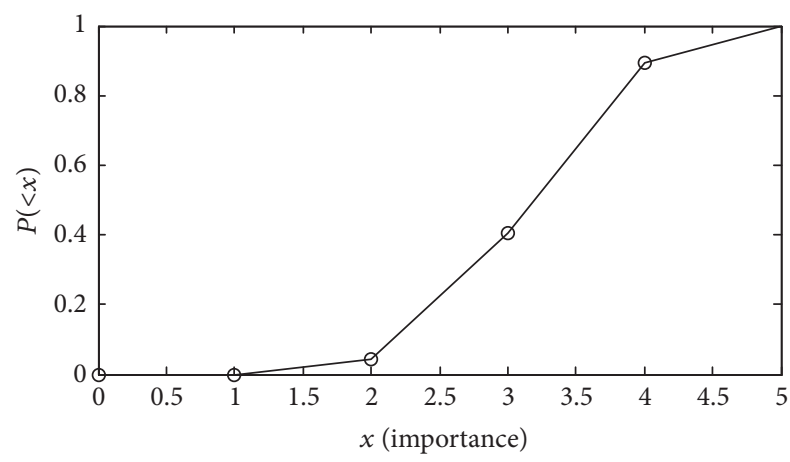

FIGURE 2: CDF for importance of "profitability" index.

Fused expert's opinions on the index "innovative development ability" could be obtained as follows:

$$
\widetilde{m}\left(C^{4}\right)=[[0.03,0.07][0.10,0.13][0.04,0.04][0.21,0.21][0.58,0.58]]
$$

CDF for its importance is shown in Figure 4.

Average degree of importance of four indices could be obtained by Monte Carlo random sampling with a sampling number of $N=2000$ times:

$$
\begin{aligned}
& u_{1}=2.06, \\
& u_{2}=3.17, \\
& u_{3}=3.69, \\
& u_{4}=1.35 .
\end{aligned}
$$

After normalization, weights of four indices "assets and solvency," "profitability," "service capability," and "innovative development ability" could be obtained, respectively. They were as follows:

$$
\begin{aligned}
& \omega_{1}=0.20, \\
& \omega_{2}=0.31,
\end{aligned}
$$

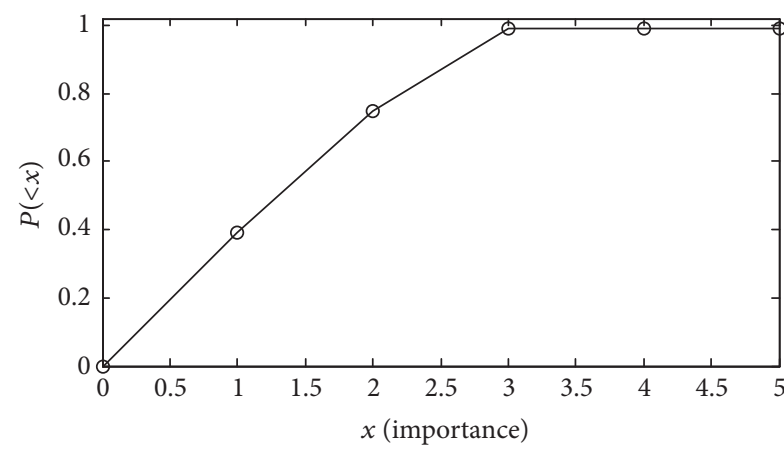

FIgURE 3: CDF for importance of "service capability" index.

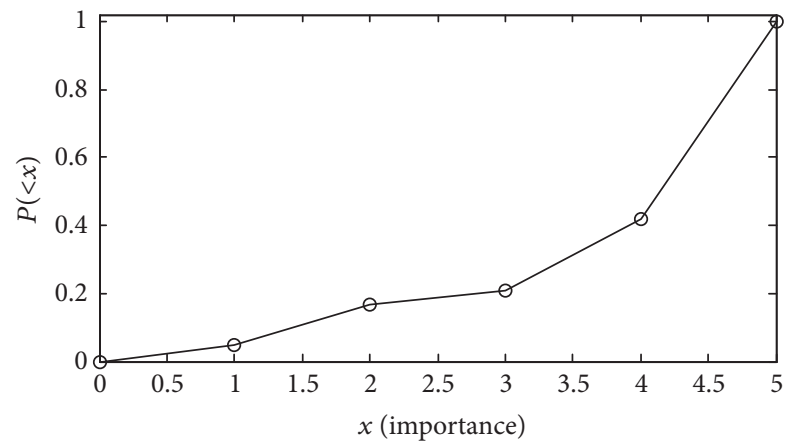

FIGURE 4: CDF for importance of "innovative development ability" index.

$$
\begin{aligned}
& \omega_{3}=0.36, \\
& \omega_{4}=0.13 .
\end{aligned}
$$

\section{Conclusions}

Index weighting is one of the key issues in group decisionmaking. Due to the complexity of the index system, experts tend to give index importance evaluation based on their own knowledge and understanding in actual group decisionmaking process. Multi-interval probability values were even assigned occasionally. In this paper, index weighting problem in such cases was studied. Various uncertain information is uniformly expressed through interval evidence structure by fully considering expert information during group decisionmaking. Then, probability information of multi-interval was fused. The importance of indices is constructed based on the 
fused information by CDF evaluating. Finally, index weights are determined by Monte Carlo random sampling.

The method proposed herein has the following advantages:

(1) It could give the weighting of group decisionmaking information with various degrees of uncertainties, such as precise evaluation information (e.g., $H_{3}(1)$ ), epistemic uncertain evaluation information (e.g., $H_{4}(0.7), H_{5}(0.3)$ ), and multi-interval probability evaluation information (e.g., $H_{3}([0.3,0.5])$, $\left.H_{4}([0.4,0.7])\right)$, which possesses good compatibility.

(2) The method draws on the idea and expression of interval evidence theory. The similarities of group decision-making information were specified by Euclidean distance between interval vectors. Weighted average method was utilized to fuse expert's information directly. Thus, the "one-vote veto" or "focusing effect" problem that arose during combination highly conflicting evidence fusion process, difficulties in constructing, and testing consistent judgment matrices by AHP could all be avoided.

(3) Digitization and visualization of the fused group decision-making information can be achieved by CDF based on importance of indices constructed according to the information fused by interval evidence theory. The subsequent random sampling process is implemented entirely by computer programs. It is simple and efficient. It owns apparent advantages for the evaluation of systems with the large index and complex targets.

\section{Conflicts of Interest}

The authors declare that they have no conflicts of interest.

\section{Acknowledgments}

This work was supported by the Scientific Research Foundation of Southwest University of Science and Technology under Grant no. 12sx7106.

\section{References}

[1] Y.-M. Wang and C. Parkan, "Multiple attribute decision making based on fuzzy preference information on alternatives: ranking and weighting," Fuzzy Sets and Systems, vol. 153, no. 3, pp. 331346, 2005.

[2] L. Jia, Z. Tong, C. Wang, and S. Li, "Aircraft combat survivability calculation based on combination weighting and multiattribute intelligent grey target decision model," Mathematical Problems in Engineering, vol. 2016, Article ID 8934749, 9 pages, 2016.

[3] Z. Pawlak and A. Skowron, "Rough sets and Boolean reasoning," Information Sciences, vol. 177, no. 1, pp. 41-73, 2007.

[4] J.-M. Wei, S.-Q. Wang, M.-Y. Wang, J.-P. You, and D.-Y. Liu, "Rough set based approach for inducing decision trees," Knowledge-Based Systems, vol. 20, no. 8, pp. 695-702, 2007.

[5] B. Meng and G. Chi, "New combined weighting model based on maximizing the difference in evaluation results and its application," Mathematical Problems in Engineering, vol. 2015, Article ID 239634, 9 pages, 2015.

[6] Y. Sun and X. Z. Bao, "A new combination weighting method and its application based on maximizing deviations," Chinese Journal of Management Science, vol. 19, no. 6, pp. 141-148, 2011.

[7] Q.-Y. Cheng, "Structure entropy weight method to confirm the weight of evaluating index," System Engineering Theory and Practice, vol. 30, no. 7, pp. 1225-1228, 2010.

[8] G. Li, "Research on method of determing combination weights based on the standard deviation revised group-G1," Journal of Systems Engineering, vol. 27, no. 1, pp. 9-18, 2012.

[9] Y. Ben-Haim, "Uncertainty, probability and information-gaps," Reliability Engineering and System Safety, vol. 85, no. 1-3, pp. 249-266, 2004.

[10] P. Sevastianov and L. Dymova, "Synthesis of fuzzy logic and Dempster-Shafer theory for the simulation of the decisionmaking process in stock trading systems," Mathematics and Computers in Simulation, vol. 80, no. 3, pp. 506-521, 2009.

[11] P. Soundappan, E. Nikolaidis, R. T. Haftka, R. Grandhi, and R. Canfield, "Comparison of evidence theory and Bayesian theory for uncertainty modeling," Reliability Engineering and System Safety, vol. 85, no. 1-3, pp. 295-311, 2004.

[12] L. Dymova and P. Sevastjanov, "An interpretation of intuitionistic fuzzy sets in terms of evidence theory: decision making aspect," Knowledge-Based Systems, vol. 23, no. 8, pp. 772-782, 2010.

[13] T. Denoeux, "Reasoning with imprecise belief structures," International Journal of Approximate Reasoning, vol. 20, no. 1, pp. 79111, 1999.

[14] T. Denoeux, "Modeling vague beliefs using fuzzy-valued belief structures," Fuzzy Sets and Systems, vol. 116, no. 2, pp. 167-199, 2000.

[15] Y.-M. Wang, J.-B. Yang, D.-L. Xu, and K.-S. Chin, "On the combination and normalization of interval-valued belief structures," Information Sciences. An International Journal, vol. 177, no. 5, pp. 1230-1247, 2007.

[16] Y.-M. Wang, J.-B. Yang, D.-L. Xu, and K.-S. Chin, “The evidential reasoning approach for multiple attribute decision analysis using interval belief degrees," European Journal of Operational Research, vol. 175, no. 1, pp. 35-66, 2006. 


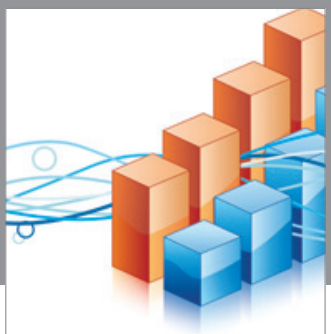

Advances in

Operations Research

vatem alat4

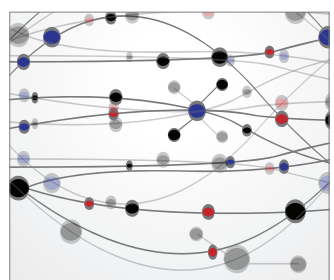

\section{The Scientific} World Journal
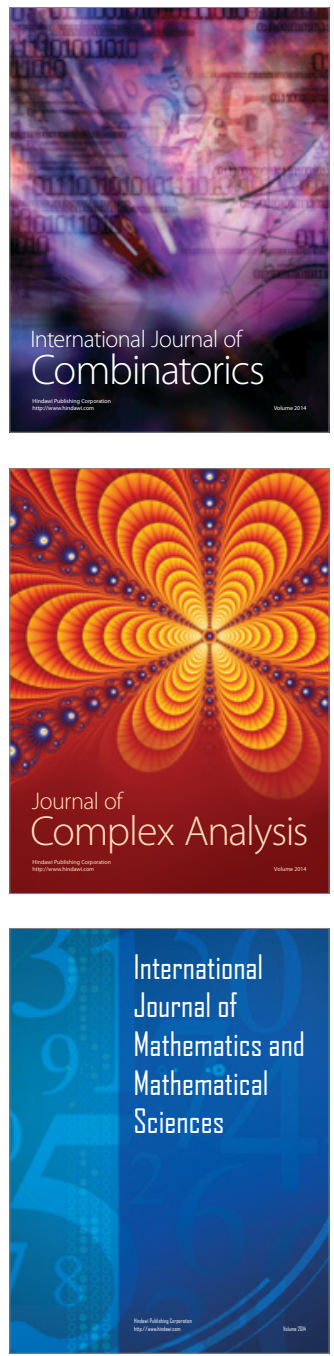
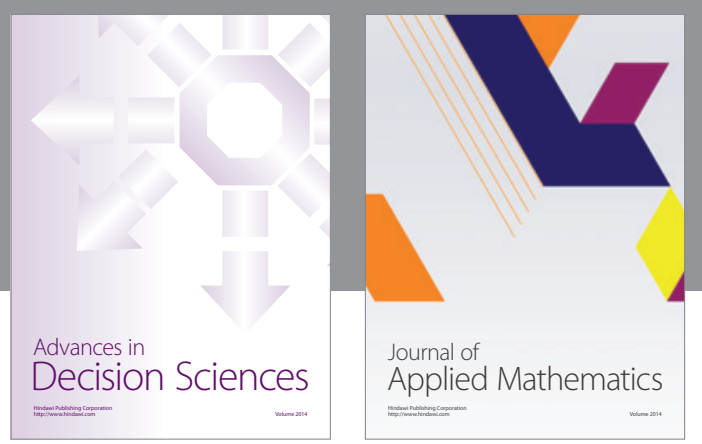

Algebra

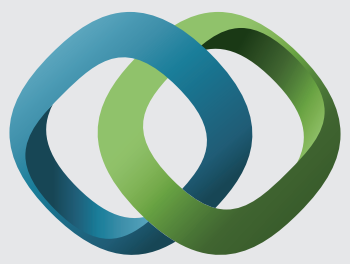

\section{Hindawi}

Submit your manuscripts at

https://www.hindawi.com
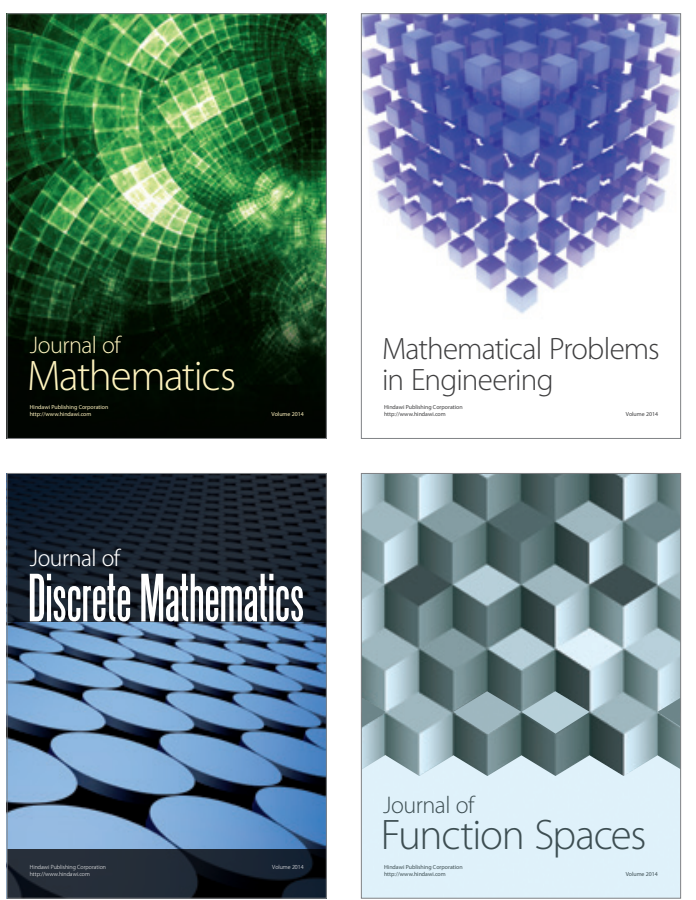

Mathematical Problems in Engineering
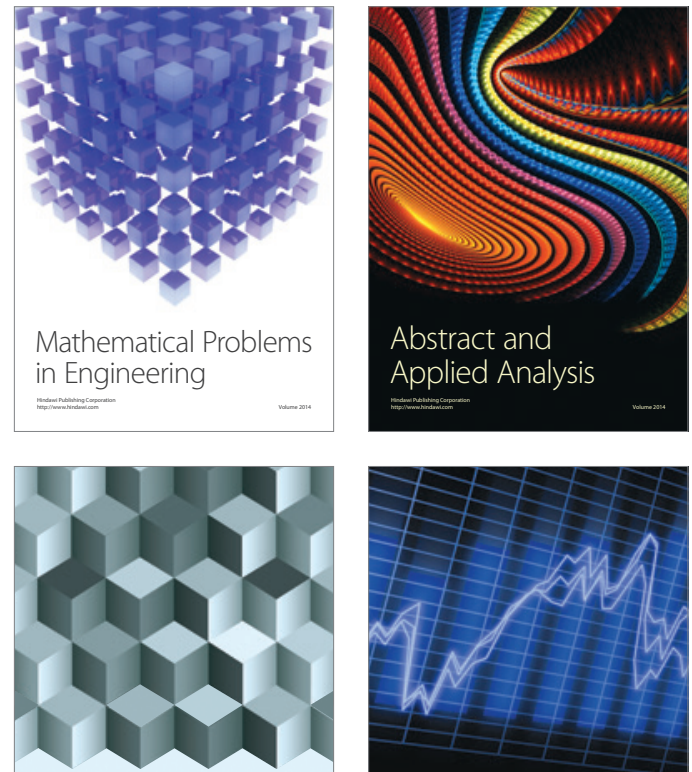

Journal of

Function Spaces

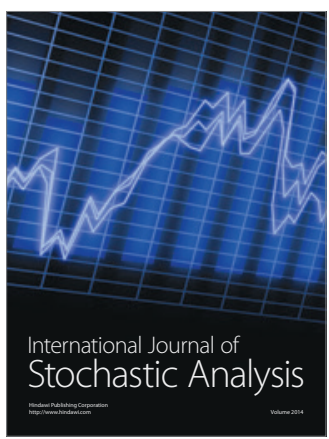

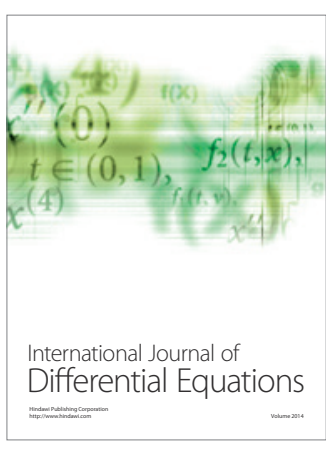
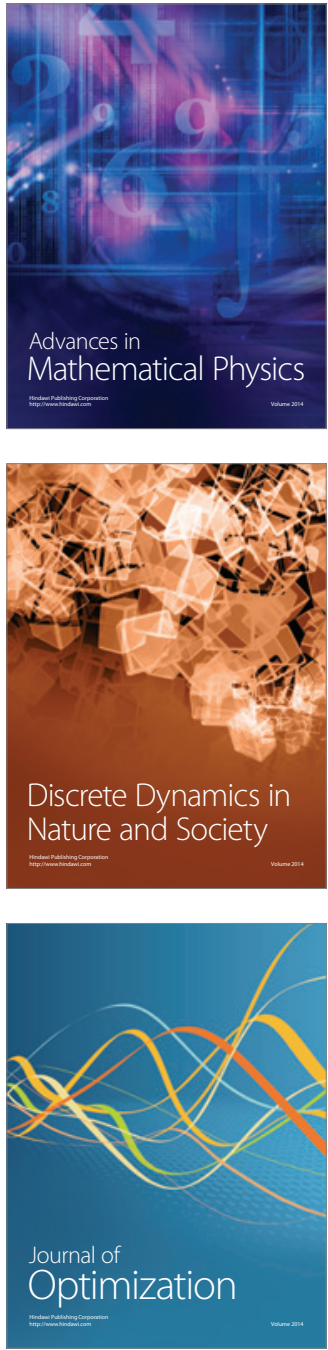